\title{
On-target off-tumor toxicity; when enhancing an NKG2D-based CAR in vitro led to severe toxicities in vivo
}

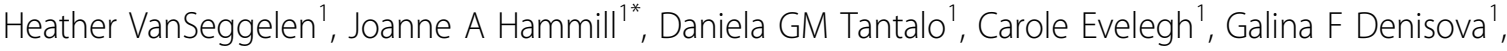 \\ Brian Rabinovich², Jacek M Kwiecien', Jonathan L Bramson \\ From Society for Immunotherapy of Cancer 29th Annual Meeting \\ National Harbor, MD, USA. 6-9 November 2014
}

Engineering $\mathrm{T}$ cells with chimeric antigen receptors (CARs) has emerged as a promising approach to adoptive $\mathrm{T}$ cell therapy for cancer. We have been studying CARs that employ the ligand-binding domain of the NKG2D receptor to target tumors. NKG2D ligand expression is increased on the surface of stressed cells, like tumor cells, making this family of ligands a target of interest for cancer immunotherapies. Two CARs were constructed: 1) a fusion of the full-length NKG2D receptor and CD3z (NKG2D $\zeta$ ) and 2) the extracellular domain of NKG2D fused to a second-generation CAR scaffold composed of transmembrane and intracellular domains from CD28 and the signaling domain of CD3z (NKG2D28 $\zeta$ ). Since surface expression of NKG2D is limited by the presence of DAP10, we also created a vector where DAP10 was co-expressed with NKG2D $\zeta$ (NKG2D $\zeta 10)$. Indeed, NKG2D $\zeta 10-C A R-T$ cells displayed a greater than 10-fold increase in CAR expression compared to NKG2D $\zeta$; NKG2D28 $\zeta$ showed an intermediate level of expression. $\mathrm{T}$ cells expressing any of the NKG2D CARs produced IFNg and TNFa in response to NKG2D ligand stimulation and efficiently killed tumor targets in vitro. However, following infusion into syngeneic hosts, we observed significant toxicity in vivo with these CAR constructs. Signs of toxicity, including poor body condition, hunched posture, labored breathing, and decreased core body temperature were observed in tumor-bearing and tumor-free mice treated with NKG2D-based CAR-T cells as compared to control mice. The severity of NKG2D CAR-T cell toxicity varied, with NKG2D $\zeta 10$ being severely toxic, NKG2D28 $\zeta$ showing intermediate toxicity, and NKG2D $\zeta$ being tolerable. Clinical symptoms of toxicity and mortality rates were exacerbated when mice received chemotherapy prior to adoptive transfer of $\mathrm{T}$ cells expressing any of the NKG2D CARs. These observations were consistent between BALB/c and C57BL/6 hosts. Further characterization revealed that the toxicity coincided with a systemic cytokine storm and lethal levels of inflammation within the lungs. These data warn that extreme caution should be taken when using NKG2D ligands for targeted immunotherapy and demonstrate that enhancing $\mathrm{T}$ cell expression of strongly activating CARs can be detrimental in vivo when the CAR target is not uniquely expressed on the tumor.

This research was supported by the CIHR and the TFRI.

\section{Authors' details}

${ }^{1}$ McMaster University, Hamilton, Ontario, Canada. ${ }^{2}$ M.D. Anderson Cancer Center, Houston, TX, USA.

Published: 6 November 2014

doi:10.1186/2051-1426-2-S3-P15

Cite this article as: VanSeggelen et al: On-target off-tumor toxicity; when enhancing an NKG2D-based CAR in vitro led to severe toxicities in vivo. Journal for ImmunoTherapy of Cancer 2014 2(Suppl 3):P15.

${ }^{1}$ McMaster University, Hamilton, Ontario, Canada

Full list of author information is available at the end of the article 